\title{
Sub-Optimal Metrics for UWB Reception in Narrowband Interference
}

\author{
Dhere, Amol
}

Published in:

IEEE 18th International Symposium on Personal, Indoor and Mobile Radio Communications, 2007. PIMRC 2007.

Link to article, DOI:

10.1109/PIMRC.2007.4394261

Publication date:

2007

Document Version

Publisher's PDF, also known as Version of record

Link back to DTU Orbit

Citation (APA):

Dhere, A. (2007). Sub-Optimal Metrics for UWB Reception in Narrowband Interference. In IEEE 18th

International Symposium on Personal, Indoor and Mobile Radio Communications, 2007. PIMRC 2007. (pp. 1-4). IEEE. https://doi.org/10.1109/PIMRC.2007.4394261

\section{General rights}

Copyright and moral rights for the publications made accessible in the public portal are retained by the authors and/or other copyright owners and it is a condition of accessing publications that users recognise and abide by the legal requirements associated with these rights.

- Users may download and print one copy of any publication from the public portal for the purpose of private study or research.

- You may not further distribute the material or use it for any profit-making activity or commercial gain

- You may freely distribute the URL identifying the publication in the public portal

If you believe that this document breaches copyright please contact us providing details, and we will remove access to the work immediately and investigate your claim 


\title{
SUB-OPTIMAL METRICS FOR UWB RECEPTION IN NARROWBAND INTERFERENCE
}

\author{
Amol Dhere \\ COM*DTU, Technical University of Denmark \\ DK 2800 Kgs. Lyngby, Denmark
}

\begin{abstract}
The performance of maximum likelihood metric for bit interleaved coded modulation scheme used in multibandOFDM depends on the variance of noise power present in each sub-carrier. It is especially heavily dependent in the presence of strong narrowband interference. In this paper two soft-metrics are proposed which are not dependent on the variance of noise power. It is seen through Monte-Carlo simulations that these metrics have good enough performance in the presence of narrowband interference.
\end{abstract}

\section{INTRODUCTION}

Wi-Media specification [1] employs multiband OFDM to provide ultra wideband communication. The coding scheme used is bit interleaved coded modulation (BICM) [2]. In which the encoded data from a convolutional encoder is interleaved and then modulated to constellation symbols through gray labelling. Modulation used is QPSK at lower data rates while at higher data rates Dual carrier modulation (DCM) is used. This paper is primarily concerned with QPSK. However the results can be applied to higher modulation also. The modulated data is then transmitted on 128 orthogonal sub-carriers spread over $528 \mathrm{MHz}$ of bandwidth by the orthogonal frequency division multiplexing (OFDM) scheme. Band hopping is used in addition to guard period to avoid inter symbol interference. Three consecutive OFDM symbols are transmitted in three different bands, thus using a total of about $1500 \mathrm{MHz}$ of bandwidth. Variable data rates are provided by puncturing the output from the rate $1 / 3$ convolution code and spreading the BICM symbols along time and frequency.

The process of encoding and decoding is as outlined in Fig. 1. As explained in [3] with BICM in HIPERLAN's using a soft decision decoding gives performance gain over hard decision decoding. Different soft decision metrics [2] [3] have been suggested for modulation schemes like 16, 64, 256 QAM etc. For QPSK, which is used in multiband OFDM, the metric is simply the distance along the two dimensions of received symbol from the symbol of interest.

In this paper the result for QPSK is first derived, and its dependence on the noise power is studied. It is seen that in the presence of coloured noise the probability of error increases drastically, if the variance of noise power is unknown in different frequency bands. Then two sub-optimal metrics are derived which are tolerant to the variance of noise power. Lastly we study the performance of these metrics for ultra wideband under narrowband interference through Monte-Carlo simulations.

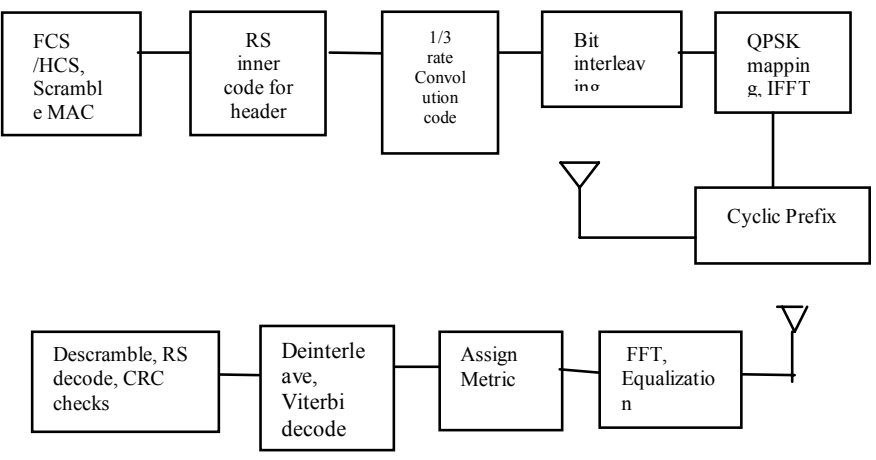

Figure 1: Multiband OFDM Tx and Rx Chain

\section{MAXIMUM LiKelihood Metric FOR QPSK}

OFDM breaks a frequency selective ultra wideband channel into several frequency non-selective narrowband channels centred on their respective sub-carriers. Although the ultra wideband channel is modelled by using gamma, SalehValenzuela distributions [4][5] the narrowband channels are assumed to be Rayleigh faded because OFDM combines several symbols to produce the received symbols on each subcarrier. Assuming coherent detection and that the guard period of OFDM symbols cancels all inter symbol interference, the symbol received on sub-carrier $n$ can be written as

$$
y_{n}=h_{n} x_{n}+z_{n}
$$

where $h_{n}$ is a rayleigh distributed coefficient for subcarrier $n$ which is assumed to be frequency non-selective and can be assumed to be estimated perfectly. $z_{n}$ is complex white gaussian noise. The effect of the channel can be cancelled by dividing the received symbol by the estimated channel coefficient. This gives

$$
r_{n}=\frac{y_{n}}{h_{n}}=x_{n}+z_{n}^{\prime}
$$

where $z_{n}{ }_{n}$ is complex Gaussian noise like $z_{n}$ but with variance scaled by $1 /\left|h_{n}\right|^{2}[3]$. The maximum likelihood (ML) metric for bit number $k$ in the label of transmitted symbol to be $b \in\{0,1\}$, considering possible transmitted symbols $x \in X$ can be written as [2] 


$$
m\left(l_{k}^{b}\right)=\log \left(\sum_{x \in X_{k}^{b}} p_{\theta}\left(y_{n} \mid x, \theta=h_{n}\right)\right)
$$

Where $X_{k}^{b}$ is the set of all symbols having the label with bit number $k$ equal to $b$. And the conditional probability is given as

$$
\begin{aligned}
& p_{\theta}\left(y_{n} \mid x, \theta=h_{n}\right)=e^{-\left|y_{n}-h_{n} x\right|^{2}} / 2 \sigma^{2}=e^{-\left|h_{n}\right|^{2}\left|r_{n}-x\right|^{2}} \\
& =e^{-\left|h_{n}\right|^{2}\left|r_{n i}-x_{i}\right|^{2} / 2 \sigma^{2}} e^{-\left|h_{n}\right|^{2}\left|r_{n j}-x_{j}\right|^{2} / 2 \sigma^{2}}
\end{aligned}
$$

The split into the real and imaginary components is assuming uncorrelated normally distributed noise power along the two dimensions. If we have inter symbol interference $x, h, y$ will be vectors. By looking at the constellation diagram for the QPSK with gray labelling in Fig. 2 the metric for $l_{k}^{b}$ can be written as

$$
\begin{aligned}
& \left.m\left(l_{1}^{0}\right)=\log \left(e^{-\left.h_{n}\right|^{2}\left(\left(r_{n i}-x_{1 i}\right)^{2}+\left(r_{n j}-x_{1 j}\right)^{2}\right)}\right) / 2 \sigma^{2}+e^{-\left|h_{n}\right|^{2}\left(\left(r_{n i}-h_{n} x_{2 i}\right)^{2}+\left(r_{n j}-x_{2 j}\right)^{2}\right)} / 2 \sigma^{2}\right) \\
& m\left(l_{1}^{0}\right)=\log \left(e^{-\left|h_{n}\right|^{2}\left(\left(r_{n i}-x_{3 i}\right)^{2}+\left(r_{n j}-x_{31 j}\right)^{2}\right)} / 2 \sigma^{2}+e^{-\left.h_{n}\right|^{2}\left(\left(r_{n i}-h_{n} x_{4 i}\right)^{2}+\left(r_{n j}-x_{4 j}\right)^{2}\right)} / 2 \sigma^{2}\right)
\end{aligned}
$$

Noting that $x_{1 i}=x_{2 i}, x_{3 i}=x_{4 i}$ and $x_{1 j}=x_{3 j}$ and $x_{2 j}=x_{4 j}$ the above expressions can be simplified as

$$
m\left(l_{1}^{0}\right)=\left|h_{n}\right|^{2}\left|r_{n_{i}}-x_{1 i}\right|^{2}, m\left(l_{1}^{1}\right)=\left|h_{n}\right|^{2}\left|r_{n i}-x_{3 i}\right|^{2}
$$

In the above expression $\sigma$, the variance of noise power is assumed to be constant across all sub-carriers and ignored. This is true if the noise is white gaussian. However for coloured noise, which is quite often the case for ultra wideband reception under narrowband interference, this assumption is not true.

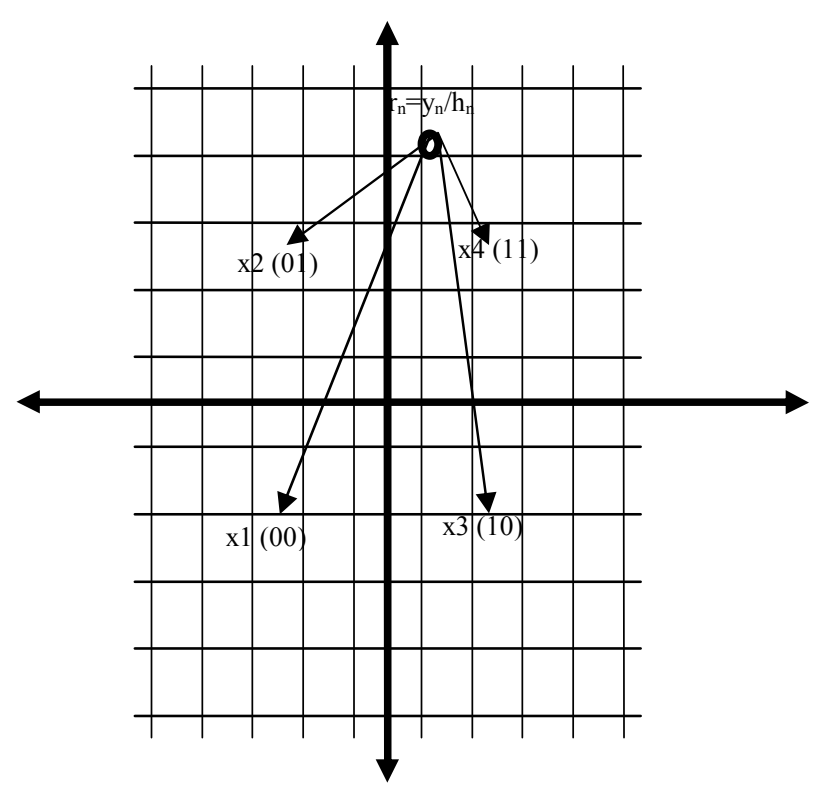

Figure 2 : QPSK constellation with Gray labeling.

\section{UWB WITH NARROWBAND INTERFERENCE}

If we take a simple case of narrowband interference effecting $n 1$ sub-carriers out of total $N$ sub-carriers, then the variance of $\mathrm{z}_{\mathrm{n}}$ in those $n l$ sub-carriers is $\sigma_{l}$ which is different from the variance $\sigma_{2}$ in other sub-carriers. Ignoring this difference in variance along the sub-carriers is equivalent to scaling the metric in the sub-carriers experiencing narrowband interference by $\sigma_{2} / \sigma_{1}$. Under the interference UWB devices are expected to function, this ratio is large and results in failure of communication. For instance if we assume that the interference bandwidth is about $50 \mathrm{MHz}$, around 10 subcarriers in a band of OFDM spectrum are affected. However because of the scaling of metric, it is observed that the performance suffers a lot to prevent any reliable communication.

Hence it is important that the variance of noise power in different sub-carriers used in one particular OFDM band be estimated and used in the metric in (3). Accurate estimation of the variance of noise power in different bands needs measurements to be done over a considerable range of time to get reliable figures. And errors in estimation results in scaling of the metric for each sub-carrier, leading to increased probability of error.

In order to counteract this, in multiband OFDM [1] the fixed frequency mode is used in place of band hopping when there is narrowband interference from some other radio in one particular band. In this mode band hopping is not done, and all OFDM symbols are transmitted in a single band which is free from narrowband interference.

In the following section, two sub-optimal metrics are proposed which work in the presence of narrowband interference due to their independence from the variance of noise power.

\section{Detection UNDER NARROWBAND INTERFERENCE}

Equation (4) can be approximated by expanding the exponential term and neglecting the higher order terms as $p_{\theta}(y \mid x, \theta=h)=e^{-|y-h x|^{2} / 2 \sigma^{2}} \approx 1-\frac{|y-h x|^{2}}{2 \sigma^{2}}$

Also by Bayes theorem

$p_{\theta}(x \mid y, \theta=h)=p_{\theta}(y \mid x, \theta=h) p(x) / p(y)$ or

$p_{\theta}(x \mid y, \theta=h)=p(x) / p(y)-\frac{|y-h x|^{2}}{2 \sigma^{2}} p(x) / p(y)$

If transmitted symbols $x$ are all equally likely then $\frac{|y-h x|^{2}}{2 \sigma^{2}}$ can be interpreted as a measure of the nonlikelihood of a symbol $x$ given received symbol $y$,. Thus a non-likelihood of $l_{k}^{b}$ can be approximated by summing $\frac{|y-h x|^{2}}{2 \sigma^{2}}$ over $x \in X_{k}^{b}$. Similarly $\frac{2 \sigma^{2}}{|y-h x|^{2}}$ can also be roughly understood to be a measure of $p_{\theta}(x \mid y, \theta=h)$. Since these are not exact measures, they are expected to perform sub-optimally. However they can be used to come up with two metrics for likelihood and non-likelihood of $l_{k}^{b}$ which are independent of variance $\sigma$. These two metrics are 
obtained by summing the likelihood/non-likelihood over $x \in X_{k}^{b}$ and dividing by summation over $x \in X$. Thus we have

$$
\begin{gathered}
m_{1}\left(l_{k}^{b}\right)=\log \frac{\sum_{x \in X_{k}^{b}} 1 /|y-h x|^{2}}{\sum_{x \in X} 1 /|y-h x|^{2}} \\
m_{2}\left(l_{k}^{b}\right)=\frac{\sum_{x \in X_{k}^{b}}|y-h x|^{2}}{\sum_{x \in X}|y-h x|^{2}}
\end{gathered}
$$

The metric $m_{2}$ is devoid of logarithm to keep the same decision rule as for metric $\mathrm{m} 1$ and the ML metric of (5). It is also to be noted that both these metrics $m_{1}$ and $m_{2}$ are generic and can be used for any modulation.

In the case of OFDM, the received symbols on each subcarrier are $r_{n}$ as given in (2). The distances used in (6),(7) can be written as $\left(\left|h_{n}\right|\left(\left|r_{n}-x\right|\right)\right)^{2}$ and the metrics can then be given as

$$
\begin{aligned}
m_{1}\left(l_{k}^{b}\right)= & \frac{\sum_{x \in X X_{k}^{b}}\left|r_{n}-x\right|^{2}}{\sum_{x \in X}\left|r_{n}-x\right|^{2}} \\
m_{2}\left(l_{k}^{b}\right)= & \frac{\sum_{x \in X_{k}^{b}} 1 /\left|r_{n}-x\right|^{2}}{\sum_{x \in X} 1 / r_{n}-\left.x\right|^{2}}
\end{aligned}
$$

These expressions enable us to save the computation of metrics, because they can now be pre-computed by quantizing the complex plain into small regions like shown in Fig 2.

\section{Performance Evaluation}

For evaluating the performance of the proposed metrics Monte-Carlo simulations were done. The encoding scheme for the transmitter side is as shown in Fig. 1. The QPSK mapped data is put on orthogonal sub-carriers by an IDFT transform to form an OFDM symbol. A cyclic prefix of length 25 is attached to this prefix to tackle inter symbol interference. No band hopping is done and all the OFDM symbols occupy the same $528 \mathrm{MHz}$ of bandwidth. The baseband signal is subjected to narrowband interference, which is generated by filtering white gaussian noise with an elliptical low pass filter of pass band $0.1 \pi$. The interference is simulated to be from an 802.11 device which occupies around $50 \mathrm{MHz}$ of bandwidth and uses OFDM. Since OFDM symbol's power spectral density is flat, we model the interference as filtered white gaussian noise.

At the decoder the received baseband data is demodulated using an IFFT to produce symbols along the different subcarriers. With the help of channel estimation sequence, the rayleigh faded taps for each of the sub-carriers is determined using MMSE algorithm. Then the effect of the channel is cancelled by dividing the received symbol by the estimated channel coefficient. The received symbol is then mapped to the quantized rectangle in which it is located, and the set of metrics for that region are picked up. These metrics are then deinterleaved, and maximum likelihood sequence estimation is then done by using the viterbi algorithm.

The UWB channel is modelled by the exponential decay model presented in [4]. Where the mean of the channel coefficients are modelled to be lognormally decaying and the coefficients are picked up from gamma distributions. The channel is assumed to be constant during the transmission of the entire packet. And for fair comparison same channel is used for all the simulations.

A packet in the simulation consists of 25 bytes of MAC/PHY header followed by 104 bytes of payload. The MAC/PHY header is protected by an inner Reed-Solomon code and has a 2 byte CRC checksum. The payload consists of 100 bytes of data followed by 4 bytes of checksum. Only if the header check passes, the payload is decoded. The bit error probability is computed from the number of packets passing both the CRC checks, as -

$$
P_{b}={\frac{N_{\text {pass }}}{N_{\text {Total }}}}^{1 / 104.8}
$$

This is with the assumption that any packet which does not pass the header CRC check will not pass the payload check either. This is a valid assumption because the header has more error protection.

The results of the simulations for the proposed metrics and the ML metric with and without narrowband interference are shown in Fig. 3 and Fig. 4 respectively. It is seen that without interference when the noise is white, the two proposed metrics perform sub-optimally. The performance of he metrics $m_{1}$ and $m_{2}$ is seen to be around 3-4 dB worse. But it is to be noted that this performance difference also includes losses due to quantization. However with coloured noise affecting some of the sub-carriers it is seen that the performance of the ML metric is extremely poor when variance of noise in different sub-carriers is not known. In fact no packets are successfully received with this metric.

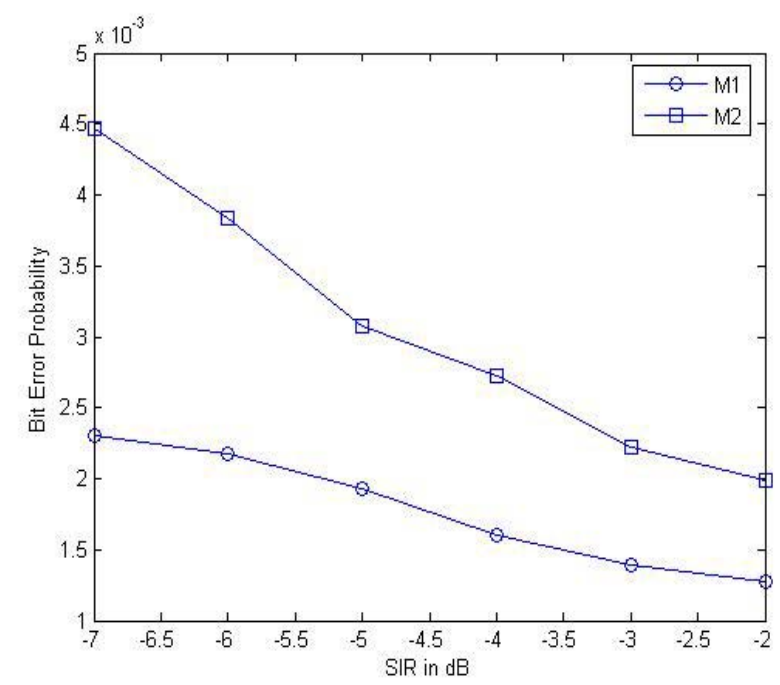

Figure 3 : Performance in narrowband interference. SNR is kept constant at $5 \mathrm{~dB}$ 


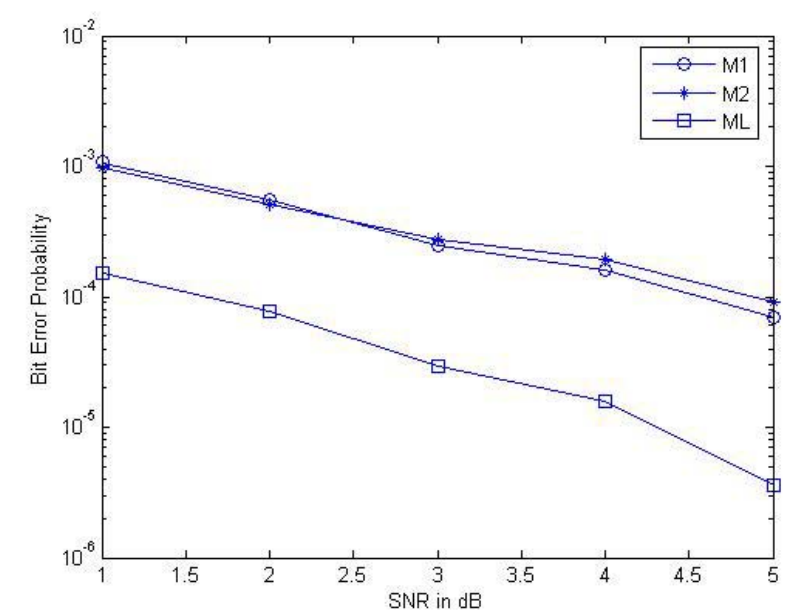

Figure 4 : Performance without interference

However with the metrics $m_{1}$ and $m_{2}$, the performance suffers, but is still good enough to receive packets. It is seen that the metric $m_{l}$ performs the best, with around $3 \mathrm{dBgain}$ over metric $m_{2}$.

\section{CONCLUSION}

The presented metrics are sub-optimal as compared to the maximum likelihood metric in the presence of white gaussian noise for OFDM transmission used in ultra wideband. However it is seen that their performance is much superior in the presence of narrowband interference where on few subcarriers the SINR is extremely poor and the variance of noise power is unknown.

\section{REFERENCES}

[1] ECMA 368 - High Rate Ultra Wideband PHY and MAC Standard,1st Edition, Dec 2005

[2] G. Caire, G. Taricco, E. Biglari, "Bit-Interleaved Coded Modulation", IEEE Teansactions on Information Theory, Vol. 44, No. 3, May 1998.

[3] F. Tosato and P. Bisaglia, "Simplified soft-output demapper for binary interleaved COFDM with application to HIPERLAN/2," Proc. IEEE ICC 2002, vol. 2, 2002, pp. 664-668.

[4] D. Cassioli, M. Z. Win, and A. F. Molisch, "The Ultra-Wide Bandwidth Indoor Channel - From Statistical Model to Simulations", IEEE JSAC, 20, 1247-1257 (2002).

[5] A. F. Molisch, J. R. Foerster, and M. Pendergrass, "Channel Models for Ultrawideband Personal Area Networks," IEEE Wireless Commun. Mag., pp. 14-21, Dec. 2003. 